\title{
Classical simulation of arbitrary quantum noise
}

\author{
Seyyed M.H. Halataei \\ Department of Physics, University of Illinois at Urbana-Champaign, \\ 1110 West Green Street, Urbana, Illinois 61801, USA
}

(Dated: Oct. 24, 2017)

\begin{abstract}
I present an explicit classical simulation of arbitrary quantum noise for quantum models in which one qubit interacts with a quantum bath. The classical model simulates the interaction of the bath and the qubit by random unitary evolutions. I show that any arbitrary quantum dynamics, including quantum dissipation, recurrence, and dephasing, can be simulated classically when one allows the unitary operators in the classical model to depend on the initial state of the system and bath. For initial mixed states of the system and non-product states of the system and bath, I demonstrate that random unitary expansion is still possible, in terms of a set of pure states.
\end{abstract}

\section{INTRODUCTION}

Entanglement is "the trait of quantum mechanics" that "enforces its entire departure from classical lines of thought", according to Schrödinger [1]. In open quantum systems, the creation of entanglement between the system and its environment is commonly stated as the root cause of decoherence [2 11]. The loss of coherence in open quantum systems, or decoherence, itself is considered to be the reason for the appearance of classical traits in quantum systems and to be connected with the quantum-to-classical transition [12, 13].

However, recently it has been demonstrated that certain types of decoherence can be simulated classically by random unitary dynamics without appealing to entanglement with an environment and the idea of transformation of information, which comes with it [14 19]. Specifically, the pure dephasing decoherence, for principal systems with twodimensional Hilbert space, and depolarizing noise, for all dimensionalities, has been simulated classically [17.

It has been also shown that some kind of random unitary dynamics, producing classical noise, is capable of retrieving coherence, quantum correlations and entanglement between the parts of a composite quantum system, such as two qubits, that are locally interacting with the classical noise [20 24].

Moreover, the effect of entanglement between a system and one of the main two types of quantum environments, the spin bath [25, 26], can be simulated by that of the other type, the oscillator bath [27, 28], in the weak- [25, 27, 29 31] and strongcoupling [32] limits of the spin bath, while the environments and their entanglement with the principal system are strikingly different in nature [25, 33].

What is the role of entanglement in quantum decoherence? And what is the distinction between quan- tum and classical noises? This paper attempts to investigate these questions further.

For a single qubit, it is well known that every doubly stochastic (or unital) channel can be represented as a random unitary channel [14, 34 36]. A doubly stochastic channel is a completely positive map on the Hilbert space of the principal system that maps the completely mixed state onto itself. A subclass of unital channels is made up of random unitary channels which are convex combinations of unitary transformations:

$$
\mathcal{E}(\rho)=\sum_{i} p_{i} U_{i} \rho U_{i}^{\dagger} \quad\left(p_{i}>0, \sum_{i} p_{i}=1\right)
$$

We extend the idea of random unitary channels to random unitary expansions by letting the unitary operators $U_{i}$ depend on the initial state of the system-plus-environment. We show that for a single qubit with initial pure states $\rho\left(t_{i}\right)$ not only doubly stochastic operations but all quantum evolutions have random unitary expansions:

$$
\rho(t)=\sum_{\alpha} p_{\alpha} U_{\alpha} \rho\left(t_{i}\right) U_{\alpha}^{\dagger}
$$

where $U_{\alpha}$ is a function of time and the initial state of the system-plus-environment. A Lebesgue integral over an infinitely uncountable set of index $\alpha$ is intended by the sum above.

For mixed initial state $\rho\left(t_{i}\right)$ of a single qubit, we show that a random unitary expansion is possible in the following sense:

$$
\rho(t)=\sum_{\alpha} p_{\alpha} U_{\alpha} \rho_{\alpha}\left(t_{i}\right) U_{\alpha}^{\dagger}
$$

where

$$
\rho\left(t_{i}\right)=\sum_{\alpha} p_{\alpha} \rho_{\alpha}\left(t_{i}\right)
$$


and $\rho_{\alpha}\left(t_{i}\right)$ are some pure states.

We construct an explicit time-continuous classical model that simulates the effect of entanglement and derives the above results. For the sake of simplicity we first introduce the classical simulation of quantum models in which the system starts in pure states and the universe (system plus environment) starts in product states. We devote Secs. IIIV to this case and describe the quantum and classical models and prove their equivalence. In Sec. V] we relax the initial-state assumption and let the system start in a mixed state and the universe in a non-product state. We show that a random unitary expansion is still possible in this case. Finally, in Sec. VI we give three examples for the case of initial pure states. The first example is the simulation of quantum recurrence in which the entropy decreases in the intermediate stage of evolution from an almost maximum value of $\ln 2$ to zero! The second example simulates pure dephasing decoherence. The last example is a simulation of amplitude damping, which could not be achieved in the previous classical models to this general extent [18, 37].

\section{QUANTUM MODEL FOR INITIAL PURE STATES}

In this section through Sec. IV we consider all quantum models with the following four properties: (1) The central system $S$ is a single qubit. (2) The qubit interacts with an arbitrary quantum bath $B$. (3) The initial state is a product state

$$
\rho_{U}\left(t_{i}\right)=\rho^{Q}\left(t_{i}\right) \otimes \rho_{B}\left(t_{i}\right)
$$

where $t_{i}$ is the initial time, $\rho_{U}$ is the density matrix of the universe (system plus bath), and $\rho^{Q}\left(t_{i}\right)$, $\rho_{B}\left(t_{i}\right)$ are the initial density matrices of the system and the bath (We use superscript $Q$ for the density matrix of the system, instead of subscript $S$, to emphasize that this density matrix is associated with the quantum model). (4) The system is initially in a pure state,

$$
\rho^{Q}\left(t_{i}\right)=\left|\Psi_{i}\right\rangle\left\langle\Psi_{i}\right|
$$

The total Hamiltonian of the above quantum models can be decomposed into three parts as usual:

$$
H_{U}=H_{S}+H_{i n t}+H_{B}
$$

where $H_{U}, H_{S}, H_{B}$ and $H_{\text {int }}$ are the Hamiltonian of the universe, the qubit, the bath, and the interaction
Hamiltonian respectively. The density matrix of the universe evolves by the evolution unitary operator

$$
U\left(t, t_{i}\right)=e^{-i H_{U}\left(t-t_{i}\right)} .
$$

where we have set $\hbar=1$. At each $t$ the density matrix of the universe is

$$
\rho_{U}(t)=U\left(t, t_{i}\right) \rho_{U}\left(t_{i}\right) U\left(t, t_{i}\right)^{\dagger} .
$$

The quantity of interest here is the reduced density matrix of the system, which can be obtained by taking the trace of $\rho_{U}(t)$ over a basis of the bath

$$
\rho^{Q}(t)=\operatorname{Tr}_{B}\left[\rho_{U}(t)\right]
$$

Choosing some basis for the Hilbert space of the qubit, one can write $\rho^{Q}(t)$ in its matrix form

$$
\rho^{Q}(t)=\left(\begin{array}{cc}
\rho_{00}^{Q}(t) & \rho_{01}^{Q}(t) \\
\rho_{10}^{Q}(t) & \rho_{11}^{Q}(t)
\end{array}\right)
$$

Since the evolution is quite arbitrary there are only a few general statements that one can make about $\rho^{Q}(t)$. Three of them are particularly useful in our discussion:

$$
\begin{aligned}
& \rho_{00}^{Q}(t), \rho_{11}^{Q}(t) \geq 0 \\
& \rho_{00}^{Q}(t)+\rho_{11}^{Q}(t)=1 \\
& \left|\rho_{10}^{Q}(t)\right| \leq \sqrt{\rho_{00}^{Q}(t) \rho_{11}^{Q}(t) .}
\end{aligned}
$$

The first two are well known. The third one can be derived from the positivity condition of the reduced density matrix, which implies $\operatorname{det}\left(\rho^{Q}\right) \geq 0$. The positivity of the reduced density matrix itself can be derived easily from the positivity of the universe density matrix. In App. A we give a proof for (14), which is finer than the positivity condition and may be used for generalization of the argument in higher dimensions. We shall use (12)-114) in constructing the classical model in the next section.

\section{CLASSICAL MODEL FOR INITIAL PURE STATES}

The classical model consists of a stochastic magnetic field, which acts on the qubit with Hamiltonian,

$$
H_{C l}(t)=\frac{1}{2} \vec{B}(t) \cdot \vec{\sigma}
$$

where $\vec{B}(t)=\left(B_{x}(t), B_{y}(t), B_{z}(t)\right)$ and $\vec{\sigma}=$ $\left(\sigma_{x}, \sigma_{y}, \sigma_{z}\right)$ are Pauli matrices. Each $B_{j}=\left(B_{j}(t)\right.$ : 
$\left.t_{i} \leq t\right)$, for $j=x, y, z$, is a random process in the standard sense [38]. That is, each $B_{j}$ is a family of random variables $B_{j}(t)$ defined on a probability space $(\Omega, \mathcal{F}, P)$ where $\Omega$ is the sample space, $\mathcal{F}$ is a set of subsets of $\Omega$, and $P$ is a probability measure on $\mathcal{F}$. By definition, for each $t$ fixed, the random variable $B_{j}(t)$ is a function from the sample space $\Omega$ to the real line: $\omega \mapsto B_{j}(t, \omega)$. Here $\omega$ are elements of $\Omega$. For each $\omega$ fixed, $\vec{B}(t, \omega)$ is a function of $t$, called the sample path (or noise history) corresponding to $\omega$, so $\omega$ 's label the sample paths.

On each sample path $\omega$, the qubit evolves from an initial state $\rho^{C l}\left(t_{i}\right)$ to state $\rho_{\omega}^{C l}(t)$ at each $t \geq t_{i}$. The initial state does not depend on $\omega$, however at any later time the state depends on $\omega$. The evolution operator for sample path $\omega$ is

$$
U_{\omega}^{C l}=\mathcal{T} \exp \left[-i \int_{t_{i}}^{t} H_{\omega}^{C l}(t) d t\right]
$$

where $\mathcal{T}$ denotes time ordering and

$$
H_{\omega}^{C l}(t)=\frac{1}{2} \vec{B}(t, \omega) \cdot \vec{\sigma}
$$

The evolved state at time $t$ can be written as

$$
\rho_{\omega}^{C l}(t)=U_{\omega}^{C l} \rho^{C l}\left(t_{i}\right) U_{\omega}^{C l \dagger} .
$$

In a classical noise model, the standard approach [17] is to consider the density matrix of the qubit at time $t$ as the expectation value of $\rho_{\omega}^{C l}(t)$ over all sample paths,

$$
\rho^{C l}(t)=\left\langle\rho_{\omega}^{C l}(t)\right\rangle_{\omega}
$$

where \langle\rangle$_{\omega}$ denotes the expectation function.

Our goal is to construct the random magnetic field process $\vec{B}(t)$ such that $\rho^{C l}(t)=\rho^{Q}(t)$ when the two models start from the same initial state $\rho^{C l}\left(t_{i}\right)=$ $\rho^{Q}\left(t_{i}\right)$.

To this end, we begin with introducing, on a probability space $(\Omega, \mathcal{F}, P)$, a random process $\Phi=(\Phi(t)$ : $\left.t_{i} \leq t\right)$ with the following properties:

1. For all $\omega \in \Omega, \Phi\left(t_{i}, \omega\right)=0$

2. For each $\omega$ fixed, $\Phi(t, \omega)$ is differentiable with respect to $t$.

3. For each $t$ fixed, the probability density function of the random variable $\Phi(t)$ is a Gaussian with mean zero and variance $\sigma^{2}(t)$,

$$
p_{\Phi}(\phi, t)=\frac{1}{\sqrt{2 \pi \sigma^{2}(t)}} \exp \left[-\frac{\phi^{2}}{2 \sigma^{2}(t)}\right] \text {. }
$$

where the probability density function $p_{\Phi}(\phi, t)$ for each $t$ is defined from the probability measure $P$ as

$$
p_{\Phi}(\phi, t)=\lim _{\epsilon \rightarrow 0} \frac{1}{\epsilon} P\{\omega: \phi \leq \Phi(t, \omega) \leq \phi+\epsilon\}
$$

4. The variance $\sigma^{2}(t)$ is

$$
\sigma^{2}(t)=-2 \lim _{s \rightarrow t} \ln \frac{\left|\rho_{10}^{Q}(s)\right|}{\sqrt{\rho_{11}^{Q}(s) \rho_{00}^{Q}(s)}}
$$

Inequality (14) guarantees that the right hand side of (22) is nonnegative. Hence, it can be considered as the variance of a Gaussian distribution. In the case that $\sigma^{2}(t)=0$ the Gaussian distribution becomes a delta function. For the case $\sigma^{2}(t)=\infty$ the distribution (20) is interpreted as a uniform distribution over the entire real line. App. B gives a construction of $\Phi(t, \omega)$ which satisfies the above properties.

By use of the probability density function (20-21), we can calculate the expectation of any function of $\Phi(t, \omega)$ for each $t$ fixed. As we shall see, it is particularly useful to calculate $\langle\exp [ \pm i \Phi(t, \omega)]\rangle_{\omega}$ :

$$
\begin{aligned}
\left\langle e^{ \pm i \Phi(t, \omega)}\right\rangle_{\omega} & =\int_{-\infty}^{\infty} e^{ \pm i \phi} P\{\omega: \phi \leq \Phi(t, \omega) \leq \phi+d \phi\} \\
& =\int_{-\infty}^{\infty} e^{ \pm i \phi} p(\phi, t) d \phi=e^{-\sigma^{2}(t) / 2} \\
& =\frac{\left|\rho_{10}^{Q}(t)\right|}{\sqrt{\rho_{11}^{Q}(t) \rho_{00}^{Q}(t)}}
\end{aligned}
$$

where the first integral above is a Lebesgue integral that is written in terms of an ordinary Reimann integral on the second line by use of the probability density function.

We define for each $\omega$ fixed, functions $a(t, \omega)$, $b(t, \omega)$ :

$$
\begin{aligned}
& a(t, \omega)=\sqrt{\rho_{00}^{Q}(t)} \\
& b(t, \omega)=\sqrt{\rho_{11}^{Q}(t)} e^{i \operatorname{Arg}\left[\rho_{10}(t)\right]} e^{i \Phi(t, \omega)}
\end{aligned}
$$

Here Arg is the argument function over complex numbers (e.g. $z=|z| e^{i \operatorname{Arg}[z]}$ ). Note that $a(t, \omega)$ is a deterministic function of $t$ and is independent of sample path $\omega$, however, $b(t, \omega)$ is a random function and depends on the sample path. Nevertheless, on each sample path one always has the identity

$$
|a(t, \omega)|^{2}+|b(t, \omega)|^{2}=\rho_{00}^{Q}(t)+\rho_{11}^{Q}(t)=1
$$


where we used Eq. (13) in the last equality.

We are now ready to give the explicit form of $\vec{B}(t, \omega)$ :

$$
\begin{aligned}
B_{z}(t, \omega) & =i\left[\dot{a}(t, \omega) a(t, \omega)^{*}+\dot{b}(t, \omega)^{*} b(t, \omega)\right] \\
B_{+}(t, \omega) & =-i\left[\dot{a}(t, \omega)^{*} b(t, \omega)-\dot{b}(t, \omega) a(t, \omega)^{*}\right] \\
B_{x}(t, \omega) & =\operatorname{Re} B_{+}(t, \omega) \\
B_{y}(t, \omega) & =\operatorname{Im} B_{+}(t, \omega)
\end{aligned}
$$

where overdots denote derivatives with respect to $t$. $B_{x}(t, \omega)$ and $B_{y}(t, \omega)$ are by definition real-valued. It is easy to show that $B_{z}(t, \omega)$ is also real-valued:

$$
\begin{aligned}
B_{z}(t, \omega)-B_{z}(t, \omega)^{*} & =i\left[\dot{a} a^{*}+\dot{a}^{*} a+\dot{b}^{*} b+\dot{b} b^{*}\right] \\
& =i \frac{\partial}{\partial t}\left[a a^{*}+b b^{*}\right]=0
\end{aligned}
$$

Here we omitted $(t, \omega)$ dependencies of $a(t, \omega)$, $b(t, \omega)$ for brevity and used (26) in the last step. Eq. (31) implies that $B_{z}(t, \omega)$ is real-valued. Thus (27)-(30) describe a well-defined stochastic magnetic field. Substituting them in Eq. (17) one obtains the Hamiltonian $H_{\omega}^{C l}(t)$ of the classical model on each sample path.

We note that the classical Hamiltonian $H_{\omega}^{C l}(t)$ depends on the total Hamiltonian of the quantum model $H_{U}$, the time elapsed from the beginning of the evolution and the initial state of the universe $\rho_{U}\left(t_{i}\right)$. This is because $a(t, \omega)$ and $b(t, \omega)$, which constitute $B(t, \omega)$, depend on $\rho^{Q}(t)$ which in turn depends on $\rho_{U}(t)$. The latter depends on $H_{U}, t$ and $\rho_{U}\left(t_{i}\right)$ (see Eqs. (7)-(11)). Thus $H_{\omega}^{C l}(t)$ not only is a function of the Hamiltonian of the quantum model, but also depends of the initial state of the universe.

\section{EQUIVALENCE OF THE QUANTUM AND CLASSICAL MODELS FOR INITIAL PURE STATES}

We assume that the qubit in the classical model starts from the same initial pure state as in the quantum model,

$$
\rho^{C l}\left(t_{i}\right)=\rho^{Q}\left(t_{i}\right)=\left|\Psi_{i}\right\rangle\left\langle\Psi_{i}\right| .
$$

Since the initial state is a pure state, on each sample path $\omega$ the qubit evolves according to the timedependent Schrödinger equation

$$
i|\dot{\Psi}(t, \omega)\rangle=H_{\omega}^{C l}(t)|\Psi(t, \omega)\rangle
$$

The solution of this Schrödinger equation on each sample path is

$$
|\Psi(t, \omega)\rangle=\left(\begin{array}{l}
a(t, \omega) \\
b(t, \omega)
\end{array}\right)
$$

as we demonstrate below: Firstly, since $\Phi\left(t_{i}, \omega\right)=0$ for all sample paths, from definitions (24)-(25) one can see that

$$
\left|\Psi\left(t_{i}, \omega\right)\right\rangle=\left|\Psi_{i}\right\rangle
$$

for each $\omega$, modulus an overall phase factor. Secondly, $|\Psi(t, \omega)\rangle$ of Eq. (34) satisfies the Schrödinger equation (33):

$$
\begin{aligned}
H_{\omega}^{C l}|\Psi(t, \omega)\rangle & =\left(\begin{array}{cc}
B_{z} & B_{+}^{*} \\
B_{+} & -B_{z}
\end{array}\right)\left(\begin{array}{l}
a \\
b
\end{array}\right) \\
& =\left(\begin{array}{c}
i \dot{a}\left[a^{*} a+b^{*} b\right] \\
i \dot{b}\left[a^{*} a+b^{*} b\right]
\end{array}\right) \\
& =\left(\begin{array}{c}
i \dot{a} \\
i \dot{b}
\end{array}\right)=i|\dot{\Psi}(t, \omega)\rangle
\end{aligned}
$$

where we used (26). Thus, $|\Psi(t, \omega)\rangle$ is the solution of (33).

The density matrix of the qubit on each sample path is then

$$
\rho_{\omega}^{C l}(t)=|\Psi(t, \omega)\rangle\langle\Psi(t, \omega)|=\left(\begin{array}{ll}
|a|^{2} & a b^{*} \\
b a^{*} & |b|^{2}
\end{array}\right)
$$

The density matrix of the classical model is the average of the density matrices of all sample paths

$$
\rho^{C l}(t)=\left\langle\rho^{C l}(t)\right\rangle_{\omega}=\left(\begin{array}{cc}
\left\langle|a|^{2}\right\rangle_{\omega} & \left\langle a b^{*}\right\rangle_{\omega} \\
\left\langle b a^{*}\right\rangle_{\omega} & \left\langle|b|^{2}\right\rangle_{\omega}
\end{array}\right) .
$$

Since $|a(t, \omega)|^{2}=\rho_{00}^{Q}(t)$ and $|b(t, \omega)|^{2}=\rho_{11}^{Q}(t)$ are deterministic functions,

$$
\begin{aligned}
& \left\langle|a(t, \omega)|^{2}\right\rangle_{\omega}=\rho_{00}^{Q}(t) \\
& \left\langle|b(t, \omega)|^{2}\right\rangle_{\omega}=\rho_{11}^{Q}(t) .
\end{aligned}
$$

For the off-diagonal term $\left\langle b a^{*}\right\rangle_{\omega}$ we have

$$
\left\langle b(t, \omega) a(t, \omega)^{*}\right\rangle_{\omega}=\sqrt{\rho_{00}(t) \rho_{11}(t)} e^{i \operatorname{Arg}\left[\rho_{10}(t)\right]}\left\langle e^{i \Phi(t, \omega)}\right\rangle_{\omega}
$$

By use of Eq. (23), the above expression simplifies to

$$
\left\langle b a^{*}\right\rangle_{\omega}=\left|\rho_{10}^{Q}(t)\right| e^{i \operatorname{Arg}\left[\rho_{10}^{Q}(t)\right]}=\rho_{10}^{Q}(t)
$$

Similarly, one obtains

$$
\left\langle a b^{*}\right\rangle_{\omega}=\left|\rho_{10}^{Q}(t)\right| e^{-i \operatorname{Arg}\left[\rho_{10}^{Q}(t)\right]}=\rho_{01}^{Q}(t) .
$$

Finally, by substituting (39), (40), (42), and (43) into (38) we obtain

$$
\rho^{C l}(t)=\left(\begin{array}{cc}
\rho_{00}^{Q}(t) & \rho_{01}^{Q}(t) \\
\rho_{10}^{Q}(t) & \rho_{11}^{Q}(t)
\end{array}\right)=\rho^{Q}(t)
$$


just as desired. Hence, the classical model simulates the quantum model exactly.

Summarizing, we built an stochastic magnetic field and hence a classical Hamiltonian for each history of noise. Then we showed that the density matrix of the qubit in this classical model at each moment of time is equal to the reduced density matrix of the quantum model at that time.

One can write Eq. (44) in a more familiar form

$$
\rho^{Q}(t)=\int_{-\infty}^{\infty} d \phi p(\phi, t) U_{\omega}^{C l}\left|\Psi_{i}\right\rangle\left\langle\Psi_{i}\right) \mid U_{\omega}^{C l^{\dagger}}
$$

where $p(\phi, t) d \phi=P\{\omega: \phi \leq \Phi(t, \omega) \leq \phi+d \phi\}$ and $U_{\omega}^{C l}=U_{\omega}^{C l}\left(t ; H_{U} ; \rho_{U}\left(t_{i}\right)\right)$. This form demonstrates that every quantum evolution of an open two dimensional system has a random unitary expansion (see Eq. (2)).

\section{QUANTUM AND CLASSICAL MODELS FOR INITIAL MIXED STATES}

The simulation of mixed states is similar to the one for pure states, apart from a few modifications that we mention below. For the quantum model, we relax conditions (3) and (4) of Sec. II for initial states of the universe and the principal system (a single qubit), and let them to start in any arbitrary states. That is the universe can start in a non-product state $\rho_{U}\left(t_{i}\right)$ and the system can start in a mixed state $\rho^{Q}\left(t_{i}\right)=\operatorname{Tr}_{B}\left[\rho_{U}\left(t_{i}\right)\right]$. The universe evolves according to the unitary evolution of Eq. (9). Relations (12)-(14) still hold since they do not depend on the initial states. Therefore we can use them in building the classical model as before.

The classical model follows the classical model of Sec. III verbatim, except we relax the first property of the random phase $\Phi(t, \omega)$ : It does not start from zero for all sample paths, rather it obeys the Gaussian distribution of Eq. (20) for all times including the initial time $t_{i}$. Thus, $\Phi(t)$ is a differentiable random process with Guassian distribution whose mean and variance are zero and $\sigma^{2}(t)$ of Eq. (22), respectively. Since the initial state is a mixed state, $\sigma\left(t_{i}\right) \neq 0$. We construct $\Phi(t, \omega)$ as in App. B. Eq. (69). Because $\sigma\left(t_{i}\right) \neq 0$, not all $\Phi\left(t_{i}, \omega\right)$ are equal to zero, as expected. The random magnetic field and the classical Hamiltonian follows Eqs. (27)-(30) and (17), as before.

To prove the equivalence of the classical model and the quantum model in the case of initial mixed state we begin with constructing wave functions $|\Psi(t, \omega)\rangle$ as in Eq. (34). By use of Eqs. (37)-(44) one can see that the density matrix of the quantum system at anytime, including the initial time $t_{i}$, can be expanded in terms of these pure states:

$$
\rho^{Q}(t)=\int_{-\infty}^{\infty} d \phi p(\phi, t)|\Psi(t, \omega)\rangle\langle\Psi(t, \omega)|
$$

One notes that wave functions $|\Psi(t, \omega)\rangle$ now do not start from the same value at $t=t_{i}$ because for two different sample paths $\omega, \omega^{\prime}$, the initial value of the random phase can be different $\Phi\left(t_{i}, \omega\right) \neq$ $\Phi\left(t_{i}, \omega^{\prime}\right)$. Nevertheless, $|\Psi(t, \omega)\rangle$ satisfy Schrödinger equation (33) on each sample path, as shown in Eq. (36). Therefore, one can obtain $|\Psi(t, \omega)\rangle$ by evolving $\left|\Psi\left(t_{i}, \omega\right)\right\rangle$ through $U_{\omega}^{C l}$ :

$$
|\Psi(t, \omega)\rangle=U_{\omega}^{C l}\left|\Psi\left(t_{i}, \omega\right)\right\rangle
$$

where $U_{\omega}^{C l}$ is defined in Eq. (16). Substituting (47) into Eq. (46) we obtain

$\rho^{Q}(t)=\int_{-\infty}^{\infty} d \phi p(\phi, t) U_{\omega}^{C l}\left|\Psi\left(t_{i}, \omega\right)\right\rangle\left\langle\Psi\left(t_{i}, \omega\right)\right| U_{\omega}^{C l^{\dagger}}$

At $t=t_{i}, U_{\omega}^{C l}$, which is a function of time, is the identity operator and Eq. (48) reduces to

$$
\rho^{Q}\left(t_{i}\right)=\int_{-\infty}^{\infty} d \phi p(\phi, t)\left|\Psi\left(t_{i}, \omega\right)\right\rangle\left\langle\Psi\left(t_{i}, \omega\right)\right| .
$$

Eqs. (48)-(49) are the random unitary expansions we intended to find for arbitrary evolution of a single qubit with initial mixed state (see Eqs. (3)-(41)). In the sense of these equations the classical model and the quantum model are equivalent for initial mixed states as well.

\section{EXAMPLES}

We consider three examples for classical simulation of quantum models. In all the examples we assume that the universe starts in the product state $\rho_{U}(0)=\left|\Psi_{i}\right\rangle\left\langle\Psi_{i}\right| \otimes \rho_{B}(0)$ where $\left|\Psi_{i}\right\rangle=\alpha|0\rangle+\beta|1\rangle$ and $\rho_{B}(0)$ will be specified for each example. We determine $\sigma^{2}(t), a(t, \omega)$ and $b(t, \omega)$ in each example. The stochastic magnetic field $B(t, \omega)$ and the classical Hamiltonian $\mathrm{H}_{\mathrm{Cl}}(t, \omega)$ can then be constructed by Eqs. (27)-(30), (17) and the discussion of Sec. 


\section{A. Quantum recurrence}

Consider a spin-boson Hamiltonian at zero temperature

$$
H_{U}=\frac{1}{2} \omega_{0} \sigma_{z}+\sigma_{z} \sum_{n=1}^{N}\left(g_{n} a_{n}^{\dagger}+g_{n}^{*} a_{n}\right)+\sum_{n=1}^{N} \omega_{n} a_{n}^{\dagger} a_{n}
$$

where $N$ is finite and the frequencies of the bath are commensurable (i.e. for each $\omega_{n}, \omega_{m}$ there are integer numbers $p_{n}, p_{m}$ such that $\left.\omega_{n} / \omega_{m}=p_{n} / p_{m}\right)$. The bath is initially in its ground state. The evolution of the reduced density matrix is then 31.

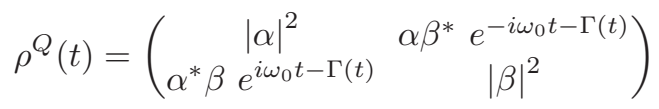

where

$$
\Gamma(t)=\sum_{n=1}^{N} 4 \frac{\left|g_{n}\right|^{2}}{\omega_{n}^{2}}\left(1-\cos \omega_{n} t\right)
$$

Since $\omega_{n}$ 's are commensurable, $\Gamma(t)$ is a periodic function. It starts at $\Gamma(0)=0$ and returns to zero with some period $P$. Between two nodes of function $\Gamma(t)$, however, the value of the function can be large if $N$ is large or coupling constants $g_{n}$ are significant. For example, for $\left|g_{n}\right|=\omega_{n}=2 \pi n / P$ and $N=30$ the average value of the function between two nodes is $\Gamma(t) \approx 120$. This gives rise to decoherence factor $\exp [-\Gamma(t)] \approx 10^{-53}$ in the off diagonal elements of (51). This implies that for $\alpha=\beta=1 / \sqrt{2}$ the system that started in a pure state with entropy $S\left[\rho^{Q}(0)\right]=\operatorname{Tr}\left[\rho^{Q}(0) \ln \rho^{Q}(0)\right]=0$ evolves to nearly the completely maxed state

$$
\rho^{Q}(t) \approx\left(\begin{array}{cc}
\frac{1}{2} & 0 \\
0 & \frac{1}{2}
\end{array}\right) \quad 0 \ll t \ll P
$$

with maximum possible entropy $S\left[\rho^{Q}(t)\right] \approx \ln 2$ for most of the times between, for example, $t=0$ and $t=P$, and then the entropy of the system decreases and the system returns to the original pure state at $t=P$ with $S\left[\rho^{Q}(P)\right]=0$.

In terms of Bloch vector the above process describes a contraction of the Bloch sphere to almost a point and then an expansion of it to its full size.

Although the entropy decreases in the intermediate stage in the above process, it can still be simulated classically. The variance $\sigma(t)^{2}$ defined in (22) is in this case

$$
\sigma^{2}(t)=2 \Gamma(t)
$$

and the functions $a(t, \omega), b(t, \omega)$ of Eqs. 24)-25 are

$a(t, \omega)=|\alpha|, \quad b(t, \omega)=|\beta| e^{i \operatorname{Arg}\left[\alpha^{*} \beta\right]} e^{i \omega_{0} t} e^{i \Phi(t, \omega)}$

where $\Phi(t, \omega)$ is the random phase defined in points 1-4 of Sec. III.

\section{B. Pure dephasing}

One can also use Hamiltonian (50) as an example of phase damping in the limit $N \rightarrow \infty$. Suppose the bath is in thermal equilibrium at some nonzero temperature $T$ and the spectral density function of the bath is ohmic: $J(\omega)=\sum_{n}\left|g_{n}\right|^{2} \delta\left(\omega-\omega_{n}\right)=$ $4^{-1} J_{0} \omega e^{-\omega / \Lambda}$, where $J_{0}$ is a dimensionless constant and $\Lambda$ is a cut-off frequency. The evolution of the reduced density matrix can be described by Eq. (51) when one substitutes for $\Gamma(t)$ the following expression 31.

$$
\begin{aligned}
\Gamma(t) & =\int_{0}^{\infty} d \omega \frac{4 J(\omega)}{\omega^{2}}(1-\cos \omega t) \operatorname{coth}\left(\omega / 2 k_{B} T\right) \\
& =\frac{J_{0}}{2} \ln \left(1+\Lambda^{2} t^{2}\right)+J_{0} \ln \left[\frac{\sinh \left(\pi k_{B} T t\right)}{\pi k_{B} T t}\right]
\end{aligned}
$$

Here $\Gamma(t)$ is an increasing function of time, which gives rise to the increase of the entropy of the system over time. There is no revival of coherence in this limit. The classical model is similar to the one in the previous subsection and is described by Eqs. (54)(55) where $\Gamma(t)$ is given by Eq. (56).

\section{Amplitude damping}

Finally, consider an amplitude damping channel [34]

$$
\rho(t)=\left(\begin{array}{cc}
1-(1-\gamma(t))\left(1-|\alpha|^{2}\right) & \alpha \beta^{*} \sqrt{1-\gamma(t)} \\
\alpha^{*} \beta \sqrt{1-\gamma(t)} & |\beta|^{2}(1-\gamma(t))
\end{array}\right)
$$

where the environment starts in the ground state and $\gamma(t)$ is the probability of decay of the qubit from the excited state to its ground state. For real physical processes, $\gamma(t)$ can be replaced by $\left(1-e^{-t / T_{1}}\right)$, where $T_{1}$ is the longitudinal relaxation time constant.

An amplitude damping channel is not a unital channel and a general classical simulation of it has not been achieved in the literature, to our knowledge. The classical model of Sec. III gives such a 
simulation by letting the unitary operators of the classical model to depend on the initial state of the universe. The ingredients of the model are as follows

$$
\begin{aligned}
a(t, \omega) & =\left[1-(1-\gamma(t))\left(1-|\alpha|^{2}\right)\right]^{1 / 2} \\
b(t, \omega) & =|\beta| \sqrt{1-\gamma(t)} e^{i \operatorname{Arg}\left[\alpha^{*} \beta\right]} e^{i \Phi(t, \omega)} \\
\sigma^{2}(t) & =\ln \left(|\alpha|^{-2}-(1-\gamma(t))\left(|\alpha|^{-2}-1\right)\right)
\end{aligned}
$$

\section{CONCLUSION}

In conclusion, we have constructed, for arbitrary quantum noises, a classical simulation of single-qubit models. We showed how entanglement between a qubit and an external bath can be modeled classically without using the bath. This was made possible by allowing the unitary operators in the classical model to depend on the initial state of the system and the bath.

We demonstrated that the reduced density matrices of quantum models that start from initial pure states have random unitary expansions. For the quantum models that start from mixed states (and even non-product states of the system and bath) we showed that the density matrices can be expressed as a random unitary expansion of some pure states.

The classical model was based on utilizing a differentiable random phase that has Gaussian distribution with time-varying variance. We gave the explicit expression for the stochastic magnetic field of the classical Hamiltonian. The field depends on the Hamiltonian of the quantum model, the time elapsed from the beginning of the evolution, and the initial state of the system and bath in the quantum model.

Simulation of quantum dissipation such as amplitude damping had not been achieved in preceding classical models, except for short times and high temperatures. Here, we offered exact results for a general simulation of such a process (amplitude damping), for arbitrary long times, as well as of quantum recurrence and pure dephasing.

Entanglement with an external environment plays an important role in quantum dissipation and decoherence of open quantum systems, beyond doubt. However, the result of this paper and its preceding counterparts show that, as far as the simulation is concerned, the distinction between quantum and classical noises may not be apparent in systems with low dimensionality.

\section{ACKNOWLEDGEMENT}

I would like to thank Mahdieh Piranaghl, Anthony J. Leggett (my PhD adviser), Bruce Hajek, Ehsan Shafiee and Richard Sawers for fruitful discussions, valuable hints and support.

\section{Appendix A A PROPERTY OF THE REDUCED DENSITY MATRIX}

In this appendix we prove the inequality (14), $\left|\rho_{10}\right| \leq \sqrt{\rho_{00} \rho_{11}}$, for any $2 \times 2$ reduced matrix.

Quite generally, the density matrix of the universe can be written in terms of a statistical mixture of pure states of the universe, $\rho_{U}=\sum_{n} p_{n}\left|\Psi_{n}\right\rangle\left\langle\Psi_{n}\right|$ where $\left|\Psi_{n}\right\rangle$ are pure states of the universe and $p_{n}$ are their statistical probabilities $\left(p_{n} \geq 0, \sum_{n} p_{n}=1\right.$.)

One can choose basis $\{|i, \mu\rangle\}$ for the universe which is a direct product of a basis of the system $\{|i\rangle\}$, where $i=0,1$, and a basis of the bath $\{|\mu\rangle\}$. Then one can expand the pure states $\left|\Psi_{n}\right\rangle$ in terms of this basis, $\left|\Psi_{n}\right\rangle=\sum_{i, \mu} c_{i, \mu}^{n}|i, \mu\rangle$ and rewrite the universe density matrix as

$$
\rho_{U}=\sum_{n ; i, j ; \mu, \nu} p_{n} c_{i, \mu}^{n} c_{j, \nu}^{n^{*}}|i, \mu\rangle\langle j, \nu|
$$

Now the reduced density matrix elements are as follows,

$$
\begin{aligned}
& \rho_{00}=\sum_{n ; \mu} p_{n}\left|c_{0, \mu}^{n}\right|^{2} \\
& \rho_{11}=\sum_{n ; \mu} p_{n}\left|c_{1, \mu}^{n}\right|^{2} \\
& \rho_{10}=\sum_{n ; \mu} p_{n} c_{1, \mu}^{n} c_{0, \mu}^{n^{*}} .
\end{aligned}
$$

$\left|\rho_{01}\right|$ satisfies the following inequality

$$
\left|\rho_{01}\right|=\left|\sum_{n ; \mu} p_{n} c_{1, \mu}^{n} c_{0, \mu}^{n^{*}}\right| \leq \sum_{n ; \mu} p_{n}\left|c_{1, \mu}^{n}\right|\left|c_{0, \mu}^{n^{*}}\right| .
$$

We define vectors $\vec{R}_{i}$

$\vec{R}_{i}=\left(\sqrt{p_{0}}\left|c_{i, 0}^{0}\right|, \sqrt{p_{0}}\left|c_{i, 1}^{0}\right|, \cdots ; \sqrt{p_{1}}\left|c_{i, 0}^{1}\right|, \sqrt{p_{1}}\left|c_{i, 1}^{1}\right|, \cdots\right)$

Then we observe that $\rho_{00}=\left|\vec{R}_{0}\right|^{2}, \rho_{11}=\left|\vec{R}_{1}\right|^{2}$, and, from inequality (65),

$$
\left|\rho_{01}\right| \leq \vec{R}_{0} \cdot \vec{R}_{1} .
$$

Since, $\vec{R}_{0} \cdot \vec{R}_{1} \leq\left|\vec{R}_{0}\right|\left|\vec{R}_{1}\right|$ we conclude that

$$
\left|\rho_{10}\right| \leq \sqrt{\rho_{00} \rho_{11}} .
$$




\section{Appendix B CONSTRUCTION OF $\Phi(t, \omega)$}

$\Phi$ is a random process with Gaussian distribution whose variance $\sigma^{2}(t)$ is time dependent. We demanded that the process be differentiable with respect to time on each sample path in order to have a well-defined magnetic field in Eqs. (27)-(30). Such a process exists, as long as $\sigma^{2}(t)$ is differentiable with respect to time [39]. There are many constructions for $\Phi$, depending on what correlation function one would like for the random process. The simplest construction is the following [39]: Let $Z$ be a standard Gaussian random variable with mean zero and variance equal to unity. By definition of random variables, $Z$ is a function from a sample space $\Omega$ to the real line : $\omega \mapsto Z(\omega)$. Now let

$$
\Phi(t, \omega)=\sigma(t) Z(\omega)
$$

where $\sigma(t)$ is taken from Eq. (22). $\Phi(t, \omega)$ has the properties we wanted. For each $t$ fixed, the distribution of the process is Gaussian because the distribution of $Z$ is Gaussian. Also the mean and variance are

$$
\begin{aligned}
& \langle\Phi(t, \omega)\rangle_{\omega}=\langle\sigma(t) Z(\omega)\rangle_{\omega}=\sigma(t)\langle Z(\omega)\rangle_{\omega}=0 \\
& \left\langle\Phi(t, \omega)^{2}\right\rangle_{\omega}=\sigma^{2}(t)\left\langle Z(\omega)^{2}\right\rangle_{\omega}=\sigma^{2}(t)
\end{aligned}
$$

as expected. For each $\omega$ fixed, $\Phi(t, \omega)$ is differentiable with respect to $t$ since $\sigma(t)$ is so. Finally, $\Phi\left(t_{i}, \omega\right)=0$ for initial pure states because $\sigma\left(t_{i}\right)=0$ for such states. In this case the right hand side of Eq. (22) is zero. For mixed states the right hand side of Eq. (22) is nonzero and $\sigma\left(t_{i}\right) \neq 0$. However, $\Phi\left(t_{i}, \omega\right)$ is not required in the classical model of Sec. V] to be zero either. Thus the random phase (69) works well for this case, too.
[1] E. Schrödinger. Discussion of probability relations between separated systems. Mathematical Proceedings of the Cambridge Philosophical Society, 31(4):555-563, 1935.

[2] Robin Blume-Kohout and Wojciech H. Zurek. Quantum darwinism in quantum brownian motion. Phys. Rev. Lett., 101:240405, Dec 2008.

[3] B. Bellomo, R. Lo Franco, and G. Compagno. Nonmarkovian effects on the dynamics of entanglement. Phys. Rev. Lett., 99:160502, Oct 2007.

[4] Hiroto Kubotani, Satoshi Adachi, and Mikito Toda. Exact formula of the distribution of schmidt eigenvalues for dynamical formation of entanglement in quantum chaos. Phys. Rev. Lett., 100:240501, Jun 2008.

[5] Thomas Gorin, Carlos Pineda, and Thomas H. Seligman. Decoherence of an $n$-qubit quantum memory. Phys. Rev. Lett., 99:240405, Dec 2007.

[6] Ángel Rivas, Susana F Huelga, and Martin B Plenio. Quantum non-markovianity: characterization, quantification and detection. Reports on Progress in Physics, 77(9):094001, 2014.

[7] Leandro Aolita, Fernando de Melo, and Luiz Davidovich. Open-system dynamics of entanglement:a key issues review. Reports on Progress in Physics, 78(4):042001, 2015.

[8] Rosario Lo Franco, Bruno Bellomo, Sabrina Maniscalco, and Giuseppe Compagno. Dynamics of quantum correlations in two-qubit systems within nonmarkovian environments. International Journal of Modern Physics B, 27(01n03):1345053, 2013.

[9] Inés de Vega and Daniel Alonso. Dynamics of nonmarkovian open quantum systems. Rev. Mod. Phys., 89:015001, Jan 2017.
[10] Ali Mortezapour, Mahdi Ahmadi Borji, and Rosario Lo Franco. Protecting entanglement by adjusting the velocities of moving qubits inside nonmarkovian environments. Laser Physics Letters, 14(5):055201, 2017.

[11] Carlos A. González-Gutiérrez, Ricardo RomáAncheyta, Diego Espitia, and Rosario Lo Franco. Relations between entanglement and purity in nonmarkovian dynamics. International Journal of Quantum Information, 14(07):1650031, 2016.

[12] Wojciech Hubert Zurek. Decoherence, einselection, and the quantum origins of the classical. Rev. Mod. Phys., 75:715-775, May 2003.

[13] Erich Joos, H Dieter Zeh, Claus Kiefer, Domenico JW Giulini, Joachim Kupsch, and Ion-Olimpiu Stamatescu. Decoherence and the appearance of a classical world in quantum theory. Springer, New York, 2003.

[14] L.J. Landau and R.F. Streater. On birkhoff's theorem for doubly stochastic completely positive maps of matrix algebras. Linear Algebra and its Applications, 193:107 - 127, 1993.

[15] Julius Helm and Walter T. Strunz. Quantum decoherence of two qubits. Phys. Rev. A, 80:042108, Oct 2009.

[16] Julius Helm, Walter T. Strunz, Stephan Rietzler, and Lars Erik Würflinger. Characterization of decoherence from an environmental perspective. Phys. Rev. A, 83:042103, Apr 2011.

[17] Daniel Crow and Robert Joynt. Classical simulation of quantum dephasing and depolarizing noise. Phys. Rev. A, 89:042123, Apr 2014.

[18] J Trapani. Stochastic noise approach to nonMarkovian decoherence in continuous variable open 
quantum systems. PhD thesis, Universita degli Studi di Milano, 2017.

[19] M. Gregoratti and R. F. Werner. Quantum lost and found. Journal of Modern Optics, 50(6-7):915-933, 2003.

[20] Rosario Lo Franco and Giuseppe Compagno. Overview on the Phenomenon of Two-Qubit Entanglement Revivals in Classical Environments, pages 367-391. Springer International Publishing, Cham, 2017.

[21] Adeline Orieux et. al. Experimental on-demand recovery of entanglement by local operations within non-markovian dynamics. Scientific Reports, 5:8575, Feb 2015.

[22] A. DArrigo, R. Lo Franco, G. Benenti, E. Paladino, and G. Falci. Recovering entanglement by local operations. Annals of Physics, 350(Supplement C):211 $-224,2014$.

[23] Bruno Leggio, Rosario Lo Franco, Diogo O. SoaresPinto, Paweł Horodecki, and Giuseppe Compagno. Distributed correlations and information flows within a hybrid multipartite quantum-classical system. Phys. Rev. A, 92:032311, Sep 2015.

[24] Jacopo Trapani, Matteo Bina, Sabrina Maniscalco, and Matteo G. A. Paris. Collapse and revival of quantum coherence for a harmonic oscillator interacting with a classical fluctuating environment. Phys. Rev. A, 91:022113, Feb 2015.

[25] N V Prokof'ev and P C E Stamp. Theory of the spin bath. Reports on Progress in Physics, 63(4):669, 2000.

[26] I. S. Tupitsyn, N. V. Prokof'ev, and P. C. E. Stamp. Effective hamiltonian in the problem of a central spin coupled to a spin environment. International Journal of Modern Physics B, 11(24):2901-2926, 1997.

[27] A.O Caldeira and A.J Leggett. Quantum tunnelling in a dissipative system. Annals of Physics, 149(2):374 - 456, 1983.

[28] A. J. Leggett, S. Chakravarty, A. T. Dorsey, Matthew P. A. Fisher, Anupam Garg, and W. Zwerger. Dynamics of the dissipative two-state system. Rev. Mod. Phys., 59:1-85, Jan 1987.

[29] A. O. Caldeira, A. H. Castro Neto, and T. Oliveira de Carvalho. Dissipative quantum systems modeled by a two-level-reservoir coupling. Phys. Rev. B, 48:13974-13976, Nov 1993.

[30] Ulrich Weiss. Quantum Disipative Systems, chapter 3, pages 65-68. Pearson Education India, 4 edition, 2012.

[31] Maximilian A Schlosshauer. Decoherence: and the quantum-to-classical transition. Springer Science \& Business Media, 2007.

[32] Seyyed MH Halataei. Mapping of the spin bath onto the oscillator bath. arXiv preprint arXiv:1703.04592, 2017.

[33] N V Prokof'ev and P C E Stamp. Giant spins and topological decoherence: a hamiltonian approach. Journal of Physics: Condensed Matter, 5(50):L663,
1993.

[34] Michael A Nielsen and Isaac Chuang. Quantum computation and quantum information. Cambridge University Press, New York, 2010.

[35] Robert Alicki and Karl Lendi. Quantum Dynamical Semigroups and Applications, volume 717 of Lecture Notes in Physics. Springer, Berlin, 2007.

[36] Erika Andersson, James D. Cresser, and Michael J. W. Hall. Finding the kraus decomposition from a master equation and vice versa. Journal of Modern Optics, 54(12):1695-1716, 2007.

[37] A. Chenu, M. Beau, J. Cao, and A. del Campo. Quantum simulation of generic many-body open system dynamics using classical noise. Phys. Rev. Lett., 118:140403, Apr 2017.

[38] Bruce Hajek. Random processes for engineers. Cambridge University Press, 2015.

[39] Bruce Hajek. private communication, 2017. 